\title{
Alkoholabhängigkeit - Internistische Komplikationen
}

\author{
S. L. Haas \\ S. V. Siegmund \\ M. V. Singer
}

\author{
Alcohol Dependence - Medical Consequences
}

\section{Zusammenfassung}

Bei der Behandlung von Alkoholabhängigen ist zu beachten, dass der langjährige chronische Alkoholabusus zu einer Vielzahl von internistischen Komplikationen führen kann. Durch die lokalen topischen Effekte des Alkohols können Entzündungen von Mund, Rachen und Speiseröhre auftreten. Aus der chronischen Entzündung kann ein maligner Tumor entstehen, wobei das Risiko durch gleichzeitigen Nikotinkonsum potenziert wird. Insbesondere hochprozentige alkoholische Getränke begünstigen die Entstehung einer Magenschleimhautentzündung, die zu einer Blutung führen kann (hämorrhagische Gastritis). Durch die Schädigung der Dünnund Dickdarmschleimhaut wird nicht nur die Resorption von Nährstoffen vermindert, sondern auch der Übertritt von Bakterienbestandteilen (Endotoxinen) in die Blutzirkulation begünstigt. Hierdurch wird die Freisetzung von Entzündungsmediatoren induziert, die zu der alkoholvermittelten Leberschädigung beiträgt. Das Schädigungsmuster der Leber weist unterschiedliche, sich überlappende Veränderungen auf, die von einer Verfettung über eine Alkoholhepatitis bis zu einer Leberzirrhose reichen können. Ungefähr $15 \%$ der Alkoholiker entwickeln eine chronische Pankreatitis, bei der das Gewebe der Bauchspeicheldrüse zerstört und durch Bindegewebe ersetzt wird. Im Spätstadium ist die Verdauung der aufgenommenen Nahrung so weit eingeschränkt, dass Durchfälle auftreten. Sind mehr als 90\% des Gewebes zerstört, resultiert über die verminderte Produktion von Insulin ein Diabetes mellitus.

\section{Abstract}

When treating patients with alcohol dependence one has to take into account that chronic alcohol abuse can lead to a variety of medical complications. Due to the direct toxic effect of alcohol inflammatory mucosal alterations of the mouth, pharynx and esophagus can be the consequence, potentially leading to malignant transformation. Concurrent smoking and alcohol misuse have a synergistic effect on the development of malignancy. Particularly alcohol beverages with a high alcohol concentration promote the development of inflammatory lessions of the gastric mucosa (hemorrhagic gastritis). In addition, mucosal damage of the small and large intestine impairs resorption of nutrients and facilitates the translocation of intracolonic bacterial components (e. g. endotoxin) into the blood circulation. Resorbed endotoxin triggers the generation of various inflammatory mediators, thus constributing to alcoholic liver disease. Alcoholic liver disease encompasses different, frequently overlapping changes, which can be divided into hepatic steatosis, alcoholic hepatitis and alcoholic liver cirrhosis. Approximately $15 \%$ of all alcohol dependent patients develop chronic pancreatitis, characterized by a destruction of the pancreatic tissue and replacement by connective tissue. In the late stage, the severe impairment of a proper digestion leads to chronic diarrhea. Via the destruction of pancreatic islet cells, diabetes results as a consequence of impaired insulin production. 
Schliuisselwörter

Alkoholabhängigkeit · Alkoholfolgeerkrankungen · Alkoholhepatitis · alkoholische Leberzirrhose · chronische Pankeatitis · Gastritis · Krebs · Tumorgenese
Key words

Alcohol dependence $\cdot$ alcohol-related organ damage · alcoholic hepatitis · alcoholic liver cirrhosis · chronic pancreatis · gastritis · steatosis · tumorgenesis

\section{Einleitung}

Durchschnittlich wurden in Deutschland im Jahr 2003 mehr als 160 Liter alkoholische Getränke pro Einwohner konsumiert. Dies entsprach einem Pro-Kopf-Verbrauch von 10,2 Litern reinem Alkohol [1]. Gleichzeitig liegt bei 1,6 Mio. Menschen eine Alkoholabhängigkeit vor; 2,6 Mio. betreiben Alkoholmissbrauch und 8 Mio. Bundesbürger konsumieren Alkohol in einem Maße, der mit einem deutlich erhöhten Risiko für Alkoholfolgeerkrankungen assoziiert ist. Dieser - auch im europäischen Vergleich - hohe Alkoholkonsum führt dazu, dass jährlich ca. 42000 Menschen an den Folgen eines übermäßigen Alkoholmissbrauchs versterben. Ursache von 17000 dieser Todesfälle ist hierbei die alkoholische Leberzirrhose [1].

Alkoholassoziierte Erkrankungen weisen unter Alkoholabhängigen eine hohe Prävalenz auf. Es ist davon auszugehen, dass $29 \%$ der Männer und 9\% der Frauen, die in ein Akutkrankenhaus eingewiesen werden, Alkoholfolgeerkrankungen aufweisen [2]. Hierbei ist zu beachten, dass alle Organsysteme bei Alkoholabhängigen pathologische Veränderungen entwickeln können, wobei neben der Höhe des Alkoholkonsums Umweltfaktoren und genetische Faktoren entscheidende Determinanten der Entstehung einer alkoholischen Organerkrankung sind.

Sowohl der ambulant tätige wie auch der in der Klinik beschäftigte Internist werden mit Erkrankungen von Oropharynx, Speiseröhre (Ösophagus), Magen, Darm, Leber und Bauchspeicheldrüse (Pankreas) als Folge eines chronischen Alkoholmissbrauchs konfrontiert [3]. 75\% aller Alkoholiker haben mindestens einmal pro Jahr Kontakt mit ihrem Hausarzt.

\section{Mund, Rachen, Speiseröhre}

Durch die lokale Wirkung von Alkohol wird die Schleimhaut von Mund, Rachen (Pharynx) und Speiseröhre (Ösophagus) geschädigt, woraus eine chronische Entzündung resultieren kann [4]. Die durch chronischen Alkoholkonsum verminderte Speichelsekretion in Verbindung mit einem Abbau von Ethanol zum toxischen Azetaldehyd durch Bakterien der Mundhöhle begünstigt die Entstehung von malignen Tumoren von Oropharynx und Ösophagus [5]. Im Gegensatz zu Ethanol gilt Azetaldehyd - das wichtigste Abbauprodukt von Ethanol - als gesicherte kanzerogene und mutagene Substanz (Abb. 1). Zu den Mechanismen der Tumorentstehung zählen die direkte Schädigung der DNA sowie die gleichzeitige Schädigung von DNA-Reparaturmechanismen [6].

Bei einem täglichen Konsum von 75 bis 100 g Alkohol (entsprechend einer Flasche Wein) steigt das Risiko, an einem Mundhöhlen- oder Kehlkopfkarzinom zu erkranken, um den Faktor 13. Durch die Schleimhautschädigung wird die Aufnahme von kanzerogenen Substanzen erleichtert, woraus zu erklären ist, dass sich durch gleichzeitigen Nikotinkonsum das Krebsrisiko potenziert. Ist das Risiko für ein Ösophaguskarzinom bei Personen mit einem täglichem Konsum von mehr als $80 \mathrm{~g}$ Alkohol 18fach erhöht, steigt das Risiko auf einen Faktor von über 44, wenn gleichzeitig täglich mehr als 20 Zigaretten geraucht werden [7]. Dies hat eine hohe klinische Relevanz, da mehr als 75\% aller Alkoholkranken einen Nikotinabusus betreiben.

Die Mehrzahl der Patienten mit einem Plattenepithelkarzinom des Ösophagus betreibt einen kombinierten schädlichen Alkohol- und Nikotinmissbrauch. Personen, die Enzymvarianten (z.B. Alkoholdehydrogenase Typ ADH-1C*1) besitzen, die zu einem beschleunigten Abbau von Alkohol zu Azetaldehyd führen, weisen ein deutlich höheres Risiko für Mund-, Rachen- und Ösophaguskarzinome auf [8].

Fallkontrollstudien konnten nachweisen, dass für alkoholinduzierte Karzinome keine Schwellendosis existiert, bis zu der auch ein moderater Alkoholkonsum zu keiner Erhöhung des Krebsrisikos führt. Dies bedeutet, dass jeder noch so niedrige Alkoholkonsum - unabhängig von den kardioprotektiven Eigenschaften - zu einer signifikanten Erhöhung des Krebsrisikos führt [9].

Neben den kanzerogenen Eigenschaften senkt Alkohol den unteren Ösphagussphinkter, der zirkulären Muskelzügen entspricht und physiologisch den Rückfluss von saurem Mageninhalt in die Speiseröhre verhindert. Hierdurch leiden Alkoholkranke gehäuft unter Sodbrennen. Die gehäuften Refluxphasen begünstigen als Folge die Entstehung von Entzündungen im Bereich des gastroösphagealen Übergangs [10]. Zusätzlich kann das bei Alkoholikern gehäufte Erbrechen zu Längseinrissen im Bereich der Schleimhaut des distalen Oesophagus (Mallory-Weiss-Läsion) und so zu akuten Blutungen führen.

\section{Magen}

Hochprozentiger Alkohol löst im Magen ein Schleimhautödem mit Entzündung aus [11-13]. Hieraus kann eine Magenschleimhautentzündung (Gastritis) resultieren, die häufig zu abdominellen Schmerzen und Inappetenz führt sowie zu einer diffusen Magenblutung. Schon die einmalige Aufnahme von hochprozentigen Alkoholika (z.B. Whisky, 40 Vol.-\%) kann eine Schleimhautentzündung auslösen, die noch nach 24 Stunden nachgewiesen werden

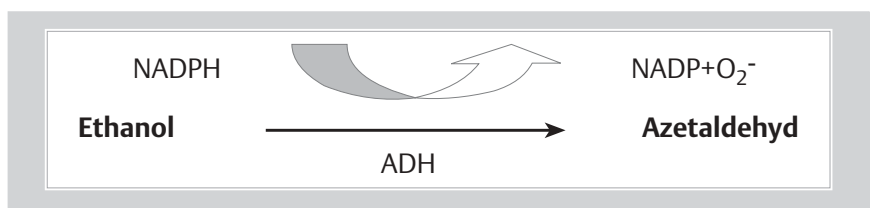

Abb. 1 Metabolismus von Ethanol über die Alkoholdehydrogenase $(\mathrm{ADH})$ zum toxischen und kanzerogenen Azetaldehyd. 
kann (Abb. 2) [11]. Diese Magenschleimhautveränderungen treten vor allem bei Alkoholikern auf, die neben Spirituosen nur wenige Mahlzeiten aufnehmen, wodurch der Alkohol ungeschützt direkt auf die Magenschleimhaut wirkt.

Entsprechend dem heutigen Kenntnisstand erhöht selbst ein hoher Alkoholkonsum weder die Inzidenz für das Magengeschwür (Ulcus ventriculi) noch für das Magenkarzinom.

Eigene Studien haben gezeigt, dass die Säuresekretion nicht durch Alkohol stimuliert wird, sondern durch Begleitstoffe (Bernsteinsäure, Maleinsäure), die bei der alkoholischen Gärung entstehen (z.B. Bier und Wein). Dagegen führen alkoholische Getränke (z.B. Whisky, Schnaps), die durch Destillation produziert werden, zu keiner signifikanten Stimulation der Magensäuresekretion [14, 15].

\section{Dünn- und Dickdarm}

Auch auf den Dünn- und den Dickdarm besitzt Alkohol eine Reihe von akuten und chronischen Effekten [16, 17]. Der schwere Alkoholexzess stimuliert die Dünndarmmotilität und kann so über eine Verkürzung der Transitzeit zu Durchfällen führen. Chronischer Alkoholkonsum schädigt die Schleimhaut von Dünn- und Dickdarm. Der oral aufgenommene Alkohol ist hierbei jedoch bereits im Magen und im oberen Dünndarm vollständig resorbiert und gelangt über die Blutzirkulation in die Schleimhaut der übrigen Dünn- und Dickdarmabschnitte, um dort seine schädigenden Einflüsse zu entfalten. Neben einer verminderten Wasserresorption wird auch die Resorption von Aminosäuren, Monosacchariden und wasserlöslichen Vitamimen (z. B. Folsäure, Vitamin B12 und Vitamin B1) vermindert. Durch die häufig bestehende unzureichende Aufnahme von Vitaminen durch die Fehlernährung des Alkoholikers wird der Vitaminmangel durch eine gleichzeitig verminderte Resorption verstärkt. Der Mangel von Vitamin B12 und Folsäure kann - neben der direkten toxischen Wirkung von Alkohol auf die Blutneubildung im Knochenmark - eine Anämie verstärken.

Durch Schädigung der Dickdarmschleimhaut wird gleichzeitig die Darmbarrierefunktion beeinträchtigt. Die intakte Schleimhautbarriere verhindert den Übertritt von Bakterien aus dem Lumen des Dickdarms in die Blutzirkulation. Bei einer Schädigung dieser Schutzfunktion gelangen auch Bestandteile der Zellwand gramnegativer Bakterien (Endotoxin bzw. Lipopolysaccharid) über die Pfortader in die Leber und induzieren dort die Freisetzung von Entzündungsmediatoren (z.B. Zytokine wie TNF $\alpha$ ) (Abb. 3) [18-21]. Diese Entzündungsreaktion spielt eine wichtige pathophysiologische Rolle bei der durch Alkohol vermittelten Leberschädigung [22].

Als gesichert gilt weiterhin der Zusammenhang von Alkohol mit dem Auftreten des malignen Enddarmtumors (Rektumkarzinom). Bei Biertrinkern, die mehr als einen Liter pro Tag $(40 \mathrm{~g} / \mathrm{d})$ trinken, ist das Risiko dreifach erhöht [23]. Der Alkohol gelangt über den Blutweg in die Darmschleimhaut, diffundiert in das Darmlumen und wird dort von Bakterien zum kanzerogenen Azetaldehyd umgewandelt. Dementsprechend finden sich bei Alkoholikern die höchsten Azetaldehydkonzentrationen im Dickdarm [24].
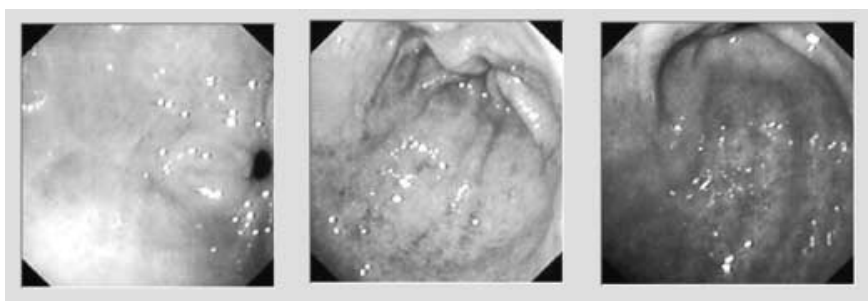

Abb. 2 Wirkung von $100 \mathrm{ml}$ Whisky auf die Magenschleimhaut. Whisky wurde endoskopisch direkt auf die Magenschleimhaut von gesunden Probanden aufgebracht (links: nach 30 Minuten, Mitte: nach 2 Stunden, rechts: nach 24 Stunden; s. [11]).

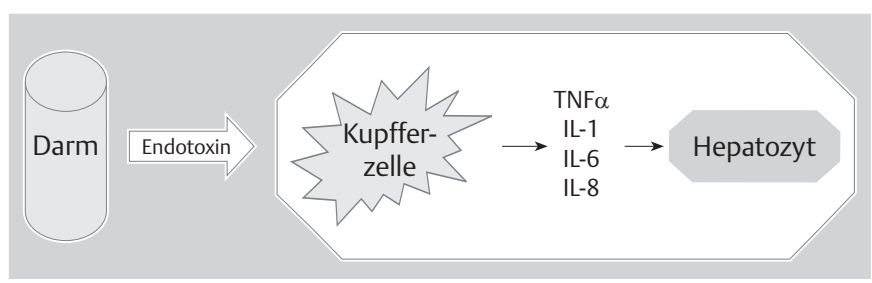

Abb. 3 Die Schädigung der Darmschleimhaut durch Alkohol begünstigt die Resorption von Endotoxin gramnegativer Bakterien. Endotoxin induziert über die Aktivierung von Hepatozyten und Kupffer-Zellen der Leber die Entzündung der Leber. Hierdurch kann eine Alkoholhepatitis und daraus resultierend eine Leberzirrhose entstehen.

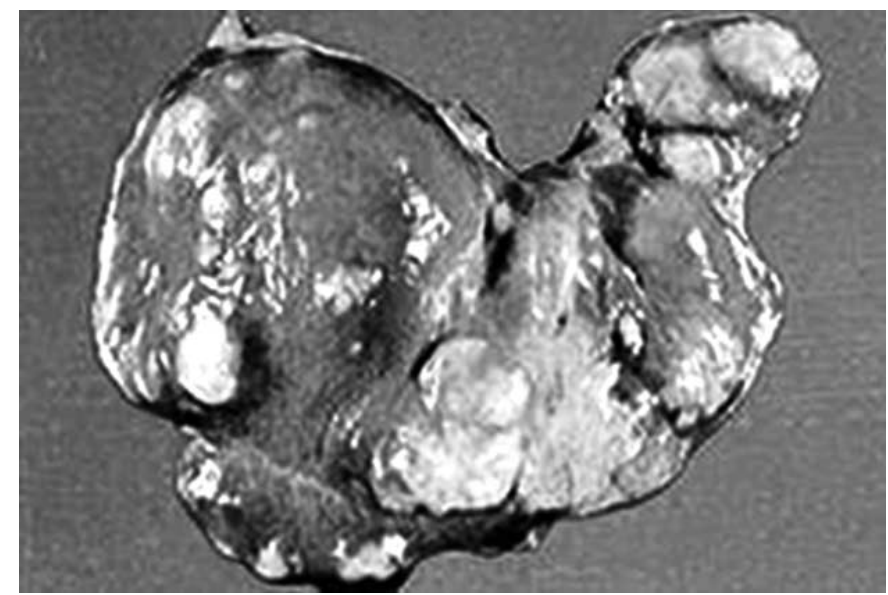

Abb. 4 Bei einer bestehenden Leberzirrhose ist das Risiko erhöht, ein Leberzellkarzinom (hepatozelluläres Karzinom) zu entwickeln. Die zirrhotisch veränderte Leber weist multiple Tumorknoten auf.

Leber

Die Leber ist eines der wichtigsten Organe, die durch hohen Alkoholkonsum geschädigt werden [25-27]. Die Veränderungen reichen hierbei von einer Fettleber (Steatosis hepatis) über eine Alkoholhepatitis bis zu einer Leberzirrhose, die mit einem erhöhtem Risiko für ein Leberzellkarzinom (hepatozelluläres Karzinom) assoziiert ist (Abb. 4). Von einer Fettleber wird gesprochen, wenn mehr als $50 \%$ der Hepatozyten vesikuläre Fetteinlagerungen aufweisen (Abb.5) [28]. Mit einer Sensitivität von über 90\% lässt sich die Fettleber mittels einer Ultraschalluntersuchung leicht nachweisen. Nur wenige Patienten mit einer Fettleber weisen Laborveränderungen (z. B. Erhöhung der $\gamma$-GT) auf. Eine kleine Gruppe der Patienten mit einer Fettleber geben Beschwerden wie ein diffuses Druckgefühl im Oberbauch an. 
Bei 15-30\% der Alkoholiker treten Veränderungen auf, die einer Alkoholhepatitis entsprechen. Im Rahmen der Entzündung finden sich in der Leber Entzündungszellen (neben hepatischen KupfferZellen und neutrophilen Granulozyten auch T-Lymphozyten), ein Untergang von Leberzellen durch Nekrose und Apoptose in Kombination mit Verfettungen und charakteristischen, jedoch nicht spezifischen Mallory-Körpern, die intrazellulären Ablagerungen von Intermediärfilamenten des Zytoskeletts entsprechen [29]. Die beginnende Fibrose beginnt um die Zentralvenen in Form eines typischen Musters (Maschendrahtfibrose) (Abb. 6) [30 - 34].

Die Beschwerden der Patienten mit Alkoholhepatitis sind sehr variabel und können von vollkommener Beschwerdefreiheit bis zu schwersten Krankheitssymptomen führen. Die durchschnittliche 30-Tage-Mortalität bei hospitalisierten Patienten liegt bei $15 \%$, kann aber in schweren Fällen bis zu $50 \%$ betragen. Auch bei vollständiger Abstinenz entwickeln zwei Drittel der Patienten mit einer schweren Alkoholhepatitis innerhalb von 18 Monaten eine Leberzirrhose.

Da zwischen allen drei Formen der alkoholischen Leberschädigung (Steatose/Alkoholhepatitis/Leberzirrhose) Überlappungen vorkommen, ist zu beachten, dass die hohe Mortalität insbesondere bei denjenigen Patienten auftritt, die bereits eine Leberzirrhose entwickelt haben.

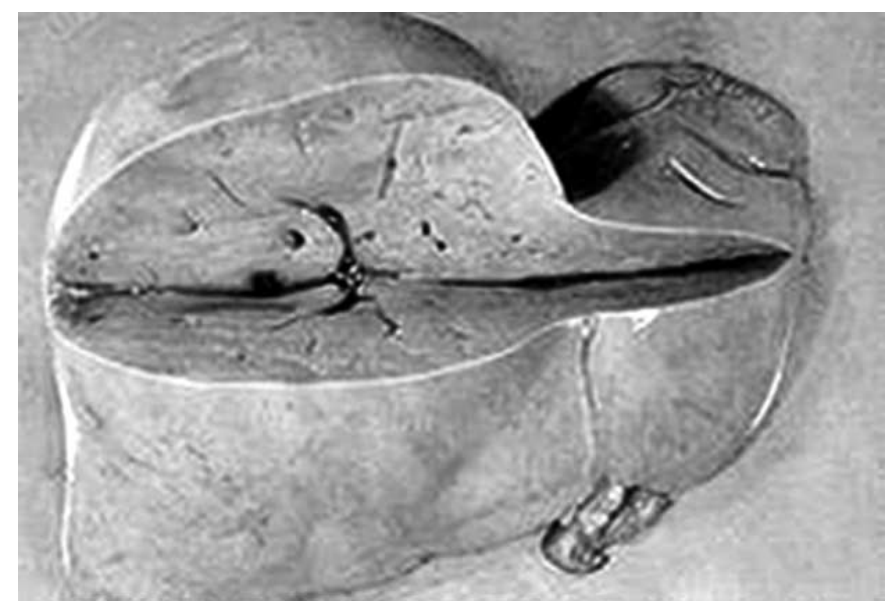

Abb. 5 Eine vergrößerte und durch die Fetteinlagerung hell erscheinende Fettleber.

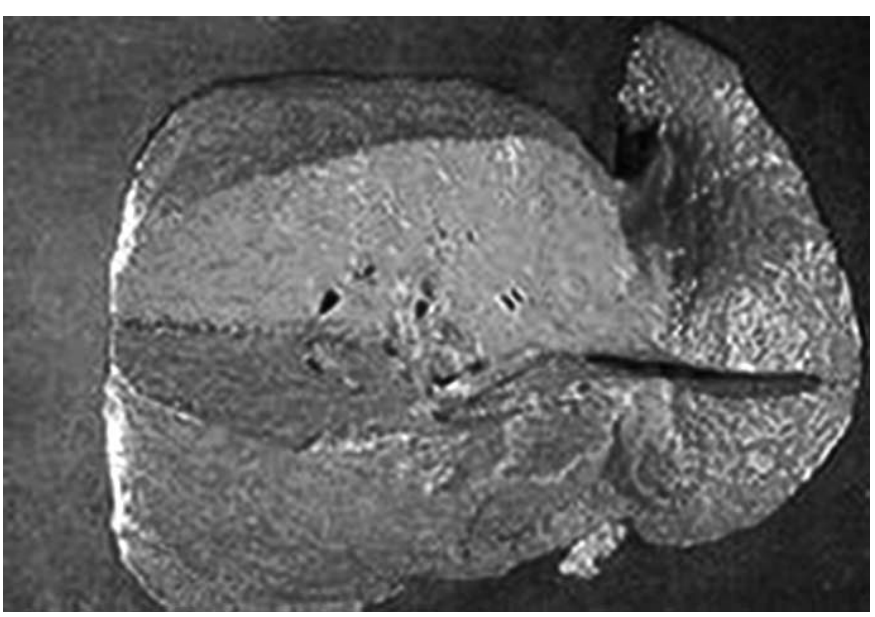

Abb. 6 Darstellung einer Leberfibrose, die durch eine vermehrte Synthese von Bindegewebe charakterisiert ist.
Die alkoholische Leberzirrhose ist durch eine vollständige Zerstörung der Leberarchitektur mit Untergang des Großteils der Leberzellen charakterisiert, die durch ein bindegewebiges Narbengewebe ersetzt sind (Abb. 6 und 7). Die Hauptfunktionen der Leber sind im fortgeschrittenen Stadium der Leberzirrhose so weit eingeschränkt, dass zahlreiche Komplikationen auftreten [35]. Mit 45\% repräsentiert der Alkoholabusus die häufigste Ursache für die Entstehung der Leberzirrhose. Die Tatsache, dass nur ca. 15\% aller Alkoholkranken eine Leberzirrhose entwickeln, legt nahe, dass bisher unzureichend definierte genetische Faktoren neben dem Alkohol und anderen Umweltfaktoren an der Entstehung der Leberzirrhose beteiligt sind.

Aus der eingeschränkten Entgiftungsfunktion resultierend treten eine Müdigkeit und Schläfrigkeit auf (hepatische Enzephalopathie). Die reduzierte Neusynthese von Proteinen in der Leber führt weiterhin zu einer verminderten Synthese von Blutgerinnungsfaktoren. Als Folge können schwere Blutungen auftreten. Ein weiteres typisches Merkmal ist die im Spätstadium auftretende Flüssigkeitsansammlung im Bauchraum (Aszites), die zu

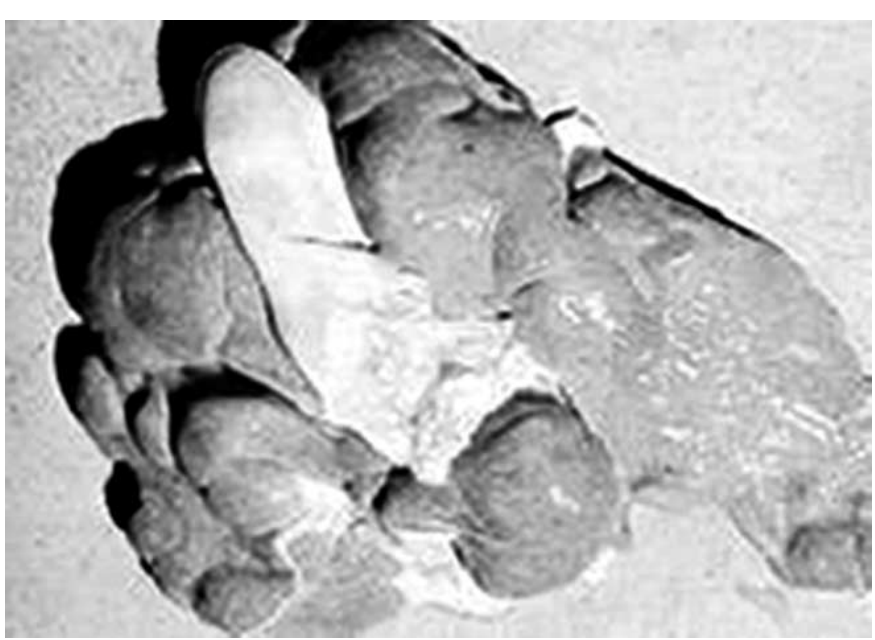

Abb. 7 Bei der Leberzirrhose ist die Leberarchitektur zerstört. Neben einem hohen Bindegewebsanteil finden sich so genannte Regeneratknoten.

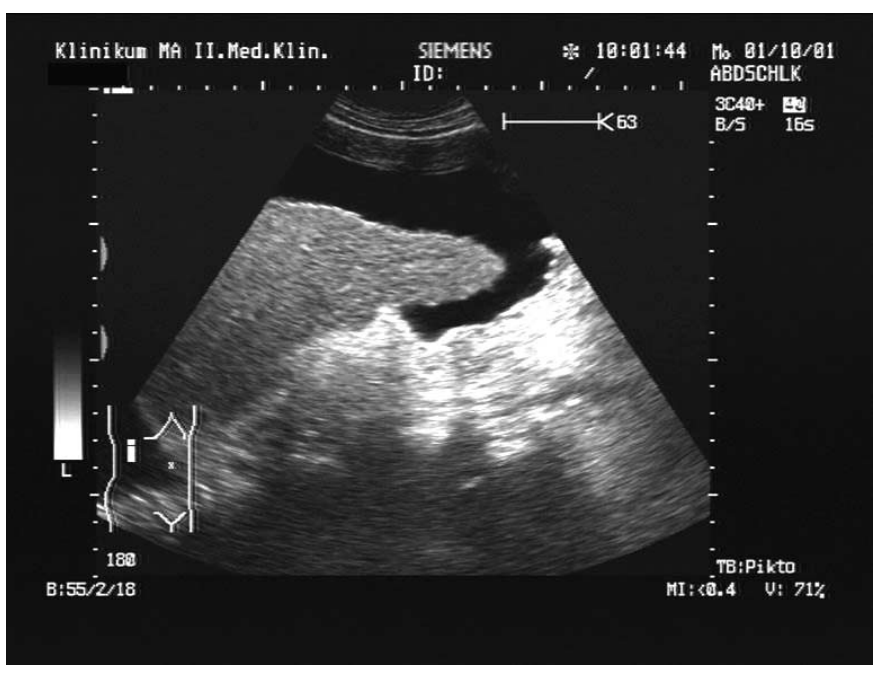

Abb. 8 Mittels Ultraschall nachgewiesene alkoholische Leberzirrhose. Die Leber ist vekleinert, hat ein inhomogenes Binnenecho und eine höckrige Oberfläche. 
einer deutlichen Einschränkung der Lebensqualität des Patienten führt und häufig wiederholte Entlastungspunktionen erforderlich macht.

Der erhöhte Gefäßwiderstand der zur Leber führenden Gefäße begünstigt die Entstehung von varikösen Erweiterungen von Venen im Bereich des Ösophagus (Ösophagusvarizen), die bei Ruptur zu lebensbedrohlichen Blutungen führen können [36].

Aufgrund der hohen Prävalenz der alkoholischen Leberzirrhose repräsentiert diese mit der chronischen viralen Hepatitis die häufigste Ursache für eine Lebertransplantation in den westlichen Industrienationen. Studien der vergangenen Jahre haben zeigen können, dass bei Patienten mit einer chronischen Hepatitis $C$ selbst bei geringem Alkoholkonsum das Fortschreiten der Leberschädigung deutlich beschleunigt wird. Gleichzeitig ist zu beachten, dass die Prävalenz der chronischen Hepatitis $C$ bei Alkoholikern im Vergleich zur Normalbevölkerung deutlich erhöht ist [37, 38].

\section{Bauchspeicheldrüse}

Ein langjähriger Alkoholmissbrauch kann zu einer akuten oder chronischen Entzündung der Bauchspeicheldrüse führen (akute und chronische Pankreatitis) [39, 40]. Es wird angenommen, dass bis zu 15\% der Alkoholiker eine chronische Pankreatitis entwickeln [41]. Langzeitstudien haben nachgewiesen, dass bei fortgesetzt hohem Alkoholkonsum bei Männern nach durchschnittlich 17 Jahren und bei Frauen nach 10 Jahren eine chronische Pankreatitis auftritt [42]. Wiederkehrende Entzündungsschübe führen zu einer progredienten Zerstörung des Organs mit Ausbildung von bindegewebigem Narbengewebe. Die akuten Schübe sind in der Regel mit heftigen abdominellen Schmerzen verbunden, wobei der klinische Verlauf variabel ist. Schwere Verläufe können trotz maximaler intensivmedizinischer Therapie letal verlaufen. Post-Mortem-Studien haben gezeigt, dass bei $47 \%$ der Patienten, die an einer „akuten Pankreatitis“ verstarben, bereits fortgeschrittene histologische Veränderungen des Pankreas vorlagen, die einer chronischen Pankreatitis entsprachen.

Bei der weit fortgeschrittenen Zerstörung des Pankreas ist die Funktion der Bauchspeicheldrüse so weit kompromittiert, dass die Enzymsynthese und -freisetzung hochgradig eingeschränkt sind, sodass der enzymatische Abbau insbesondere der aufgenommenen Fette nicht mehr suffizient erfolgt. Durch den hochgradig eingeschränkten Abbau von Fett treten abdominelle Schmerzen mit Durchfällen auf. In diesen Fällen kann die orale Gabe von Pankreasenzymen, die aus Schweinepankreas gewonnen wurden, die Beschwerden lindern. Bei Verlust von mehr als $90 \%$ der Inselzellen des Pankreas tritt ein so genannter pankreopriver Diabetes mellitus auf. In aller Regel sind Insulingaben erforderlich, um eine ausgeglichene Glukosehomöostase zu ermöglichen.

Wie auch die Leberzirrhose ein erhöhtes Risiko für die Entstehung eines Leberzellkarzinoms besitzt, geht auch die chronische Pankreatitis mit einem erhöhten Tumorrisiko einher. Das Pankreaskarzinom ist auf dem Boden einer chronischen Pankreatitis im Frühstadium häufig nur schwer zu diagnostizieren und weist im Vergleich zu anderen Tumoren eine äußerst hohe Letalität auf (5-Jahres-Mortalität > 95\%) [43].

\section{Schlussfolgerung}

Hoher fortgesetzter Alkoholkonsum führt über die Schädigung von Ösophagus, Magen, Dünn- und Dickdarm, Leber und Pankreas zu zahlreichen internistischen Komplikationen (Tab.1). Die Mechanismen und Faktoren, die bestimmen, weshalb nur ein Teil der Alkoholiker spezifische Organschäden entwickelt, ist

Tab. 1 Internistische Komplikationen der Alkoholabhängigkeit

- Entzündung von Speiseröhre und Magen (Ösophagitits, Gastritis)
- Krebs von Mund, Rachen und Speiseröhre (Plattenepithelkarzinome)
- Sodbrennen (durch Senkung des unteren Ösophagusspinkters)
- verminderte Resorption von Monosacchariden, Aminosäuren und Vitami-
nen (Vitamin B12, Folsäure, Vitamin B1) im Dünndarm
- Durchfall (bei Alkoholexzess oder chronischer Pankreatitis)
- Karzinom des Enddarms (Rektumkarzinom)
- akute und chronische Entzündung der Bauchspeicheldrüse (akute und
chronische Pankreatitis)
- Fettleber, Alkoholhepatitis und Leberzirrhose

nur unzureichend bekannt und Inhalt der aktuellen Forschung [44]. Forschungsergebnisse der vergangenen Jahre weisen hierbei auf individuelle genetische Risikofaktoren als entscheidende Determinanten hin.

\section{Literatur}

${ }^{1}$ Merfert-Diete C. Zahlen und Fakten in Kürze. In: Deutsche Hauptstelle für Suchtfragen (Hrsg). Jahrbuch Sucht 2003. Geesthacht: NeulandVerlag, 2003: 8-17

${ }^{2}$ Gerke P, Hapke U, Rumpf HJ et al. Alcohol-related diseases in general hospital patients. Alcohol Alcohol 1997; 32: 179-184

${ }^{3}$ Haas SL, Schneider A, Singer MV. Alkohol-assoziierte Erkrankungen. Internist (Berl) 2004; 45: 1199-1204

${ }^{4}$ Harder H, Teyssen S, Singer MV. Alkohol und Ösophagus. In: Singer MV, Teyssen S (Hrsg). Alkohol und Alkoholfolgeerkrankungen. Grundlagen - Diagnostik - Therapie. Heidelberg: Springer, 2005: 164-173

${ }^{5}$ Homan N, Jousimies-Somer H, Jokelainen K et al. High acetaldehyde levels in saliva after ethanol consumption : methological aspects.and pathogenetic implications. Carcinogenesis 1997; 18: 1739-1743

${ }^{6}$ Wang M, McIntee EJ, Cheg G et al. Identification of DNA adducts of acetaldehyde. Chem Res Toxicol 2000; 13: 1149-1157

${ }^{7}$ Tuyns AJ, Pequignot G, Jensen OM. Esophagel cancer in Ille-et-Vilaline in relation to levels of alcohol and tobacco consumption. Risks are multiplying. Bull Cancer 1977; 64: 45-60

${ }^{8}$ Visapää JP, Götte K, Benesova M et al. Increased cancer risk in heavy drinkers withthe alcohol dehydrogenase $1 C^{*} 1$ allele, possibly due to salivary acetaldehyde. Gut 2004; 53: 871 - 876

${ }^{9}$ Teschke R, Göke R. Alkohol und Krebs. In: Singer MV, Teyssen S (Hrsg). Alkohol und Alkoholfolgeerkrakungen. Gundlagen - Diagnostik - Therapie. Heidelberg: Springer, 2005: 349-363

10 Siegmund SV, Singer MV. Wirkungen von Alkohol auf den oberen Gastrointestinaltrakt und das Pankreas - Eine aktuelle Übersicht. Z Gastroenterol 2005; 43: 723-736

${ }^{11}$ Knoll MR, Kölbel CB, Teyssen S et al. Action of pure ethanol and some alcoholic beverages on the gastric mucosa in healthy humans: a descriptive endoscopic study. Endoscopy 1998; 30: 293-301 
${ }^{12}$ Harder H, Teyssen S, Singer MV. Alkohol und Magen. In: Singer MV, Teyssen S (Hrsg). Alkohol und Alkoholfolgeerkrakungen. Gundlagen Diagnostik - Therapie. Heidelberg: Springer, 2005: 174-192

${ }^{13}$ Teyssen S, Singer MV. Alcohol-related diseases of the oesophagus and stomach. Best Pract Res Clin Gastroenterol 2003; 17: 557-573

14 Teyssen S, Gonzalez-Calero G, Schimiczek M et al. Maleic acid and succinic acid in fermented alcoholic beverages are the stimulants of gastric acid secretion. J Clin Invest 1999; 103: 707 - 713

15 Teyssen S, Lenzing T, Gonzalez-Calero G et al. Alcoholic beverages produced by alcoholic fermentation but not by distillation are powerful stimulants of gastric acid secretion in humans. Gut 1997; 40: 49-56

${ }^{16}$ Bode C, Bode JC. Effect of alcohol consumption on the gut. Best Pract Res Clin Gastroenterol 2003; 17: 575-592

${ }^{17}$ Bode C, Parlesak A, Bode JC. Alkohol und Darm. In: Singer MV, Teyssen S (Hrsg). Alkohol und Alkoholfolgeerkrakungen. Gundlagen - Diagnostik - Therapie. Heidelberg: Springer, 2005: 193-202

${ }^{18}$ Hoek JB, Pastorino JG. Ethanol, oxidative stress, and cytokine-induced liver cell injury. Alcohol 2002; 27: 63-68

19 Jaeschke H, Smith CW, Clemens MG et al. Mechanisms of inflammatory liver injury: adhesion molecules and cytotoxicity of neutrophils. Toxicol Appl Pharmacol 1996; 139: 213-226

${ }^{20} \mathrm{McClain}$ CJ, Barve S, Deaciuc I et al. Cytokines in alcoholic liver disease. Semin Liver Dis 1999; 19: 205 -219

${ }^{21}$ Thurman RG. II. Alcoholic liver injury involves activation of Kupffer cells by endotoxin. Am J Physiol 1998; 275: 605-611

22 Rao RK, Seth A, Sheth P. Recent Advances in Alcoholic Liver Disease I. Role of intestinal permeability and endotoxemia in alcoholic liver disease. Am J Physiol Gastrointest Liver Physiol 2004; 286: G881 -4

${ }^{23} \mathrm{Wu}$ AH, Paganini-Hill A, Ross RK et al. Alcohol, physical activity and other risk factors for colorectal cancer: a prospective study. Br J Cancer 1987; 55: 687-694

${ }^{24}$ Seitz HK, Matsuzaki S, Yokoyama A et al. Alcohol and cancer. Alcohol Clin Exp Res 2001; 25: 137S-143S

${ }^{25}$ Schäfer C, Bode JC. Epidemiologie, Pathologie und klinisches Spektrum alkoholischer Lebererkrankungen. In: Singer MV, Teyssen S (Hrsg). Alkohol und Alkoholfolgeerkrakungen. Gundlagen - Diagnostik - Therapie. Heidelberg: Springer, 2005: 257-264

${ }^{26}$ Arteel G, Marsano L, Mendez C et al. Advances in alcoholic liver disease. Best Pract Res Clin Gastroenterol 2003; 17: 625 - 647

27 Tsukamoto H, Lu SC. Current concepts in the pathogenesis of alcoholic liver injury. Faseb J 2001; 15: 1335-1349

${ }^{28}$ Day CP, James OF. Hepatic steatosis: innocent bystander or guilty party? Hepatology 1998; 27: 1463-1466
${ }^{29}$ Goodman Z, Ishak K. Hepatic histopathology. In: Schiff E, Sorrell MF, Maddrey WC (Hrsg). Schiff's diseases of the liver. Vol 1. Philadelphia: Lippincott-Raven Publishers, 1999: 53-117

${ }^{30}$ Brenner DA, Waterboer T, Choi SK et al. New aspects of hepatic fibrosis. J Hepatol 2000; 32: $32-38$

${ }^{31}$ Diehl A, Rai R. Liver regeneration. In: Schiff E, Sorrell MF, Maddrey WC (Hrsg). Schiff's diseases of the liver. Vol 1. Philadelphia: Lippincott-Raven Publishers, 1999: 39-52

${ }^{32}$ Gressner AM, Schuppan D. Cellular and molecular pathobiology, pharmacological intervention, and biochemical assessment of liver fibrosis. In: Bircher J, Benhamou JP, McIntyre N, Rizzetto M, Rodes J (Hrsg). Oxford textbook of clinical hepatology. Vol 1. Oxford, New York: Oxford University Press, 1999: 607-627

${ }^{33}$ Friedman SL. Hepatic fibrosis. In: Schiff E, Sorrell MF, Maddrey WC (Hrsg). Schiff's diseases of the liver. Philadelphia: Lippincott-Raven Publishers, 1999: 371 - 385

${ }^{34}$ Friedman SL. Liver fibrosis - from bench to bedside. J Hepatol 2003; 38 (Suppl 1): S38-53

${ }^{35}$ Lieber CS. Ethanol metabolism, cirrhosis and alcoholism. Clin Chim Acta 1997; 257: 59-84

${ }^{36}$ Bosch J, Navasa M, Garcia-Pagan JC et al. Portal hypertension. Med Clin North Am 1989; 73: 931 - 953

37 Bhattacharya R, Shuhart MC. Hepatitis C and alcohol: interactions, outcomes, and implications. J Clin Gastroenterol 2003; 36: 242 - 252

${ }^{38}$ Schiff ER, Ozden N. Hepatitis C and alcohol. Alcohol Res Health 2003; 27: $232-239$

${ }^{39}$ Singer MV, Müller MK. Epidemiologie, Ätiologie und Pathogenese der chronischen Pankreatitis. In: Mössener JG, Adler G, Flösch, UR, Singer MV (Hrsg). Erkrankungen des exkretorischen Pankreas. Jena: FischerVerlag, 1995: $313-324$

${ }^{40}$ Etemad B, Whitcomb DC. Chronic pancreatitis: diagnosis, classification, and new genetic developments. Gastroenteroloy 2001; 120: $682-707$

${ }^{41}$ Haas SL, Schneider A, Singer MV. Alcohol and pancreatitis In: Pour PM (Hrsg). Toxicology of the Pancreas. Boca Raton, Florida: Taylor and Francis, 2005: $308-327$

42 Sarles H. The geographical distribution of chronic pancreatitis. In: Johnson CD, Imrie CW (Hrsg). Pancreatic disease. Progress and prospects. Heidelberg, New York: Springer Verlag, 1991: 177-184

${ }^{43}$ Haas SL. Epidemiologie des Pankreaskarzinoms. In: Löhr M, Heinemann V, Friess HM (Hrsg). Das Pankreaskarzinom - Aktuelle Diagnostik und Therapie. Bremen, London, Boston: UNI-MED Science, 2003 